\title{
Comparing star formation models with interferometric observations of the protostar NGC 1333 IRAS 4A
}

\section{Magnetohydrodynamic collapse models ${ }^{\star}$}

\author{
P. Frau $^{1}$, D. Galli ${ }^{2}$, and J. M. Girart ${ }^{1}$ \\ ${ }^{1}$ Institut de Ciències de l'Espai (CSIC-IEEC), Campus UAB, Facultat de Ciències, Torre C-5p, 08193 Bellaterra, Catalunya, Spain \\ e-mail: frau@ice.cat \\ 2 INAF-Osservatorio Astrofisico di Arcetri, Largo E. Fermi 5, 50125 Firenze, Italy \\ Received 2 August 2011 / Accepted 26 September 2011
}

\begin{abstract}
Context. Observations of dust polarized emission toward star forming regions trace the magnetic field component in the plane of the sky and provide constraints to theoretical models of cloud collapse.

Aims. We compare high-angular resolution observations of the submillimeter polarized emission of the low-mass protostellar source NGC 1333 IRAS 4A with the predictions of three different models of collapse of magnetized molecular cloud cores.

Methods. We compute the Stokes parameters for the dust emission for the three models. We then convolve the results with the instrumental response of the Submillimeter Array observation toward NGC 1333 IRAS 4A. Finally, we compare the synthetic maps with the data, varying the model parameters and orientation, and we assess the quality of the fit by a $\chi^{2}$ analysis.

Results. High-angular resolution observations of polarized dust emission can constraint the physical properties of protostars. In the case of NCC 1333 IRAS 4A, the best agreement with the data is obtained for models of collapse of clouds with mass-to-flux ratio $>2$ times the critical value, initial uniform magnetic field of strength $\sim 0.5 \mathrm{mG}$, and age of the order of a few $10^{4} \mathrm{yr}$ since the onset of collapse. Magnetic dissipation, if present, is found to occur below the resolution level of the observations. Including a previously measured temperature profile of IRAS 4A leads to a more realistic morphology and intensity distribution. We also show that ALMA has the capability of distinguishing among the three different models adopted in this work.

Conclusions. Our results are consistent with the standard theoretical scenario for the formation of low-mass stars, where clouds initially threaded by large-scale magnetic fields become unstable and collapse, trapping the field in the nascent protostar and the surrounding circumstellar disk. In the collapsing cloud, the dynamics is dominated by gravitational and magnetic forces.
\end{abstract}

Key words. magnetohydrodynamics (MHD) - polarization - stars: formation - ISM: magnetic fields ISM: individual objects: NGC 1333 IRAS 4A

\section{Introduction}

Magnetic fields play an important role in the star formation process. Molecular clouds are expected to form dense cores through a combination of loss of magnetic and turbulent support. Eventually, a molecular cloud core overcomes magnetic support ("supercritical" stage), and collapses gravitationally. The magnetic field is then pinched and strengthened in the central regions of the core, and is expected to assume an hourglass shape (Fiedler et al. 1993; Galli \& Shu 1993a,b; Nakamura \& Li 2005).

Aspherical spinning dust particles tend to align their small axis parallel to the direction of the magnetic field. Thermal emission from such elongated grains is thus partially linearly polarized, with the polarization vector perpendicular to the magnetic field. Consequently, the polarized emission is a good tracer of the magnetic field. To test the influence of magnetic fields we compare high-angular resolution observations of the polarized emission measured at submillimeter wavelengths toward the low-mass protostar NGC 1333 IRAS 4A with non-turbulent magnetohydrodynamic (MHD) models of molecular cloud cores threaded by an initial uniform magnetic field. This first step will help to $(i)$ select the best models to describe the structure and evolution of low-mass cores, and, (ii) to better understand the

* Based on observations carried out with the SMA telescope. importance of the physical processes involved in their formation and evolution. In a subsequent paper we will consider models of magnetized molecular cores formed in a turbulent environment.

The low-mass protostar IRAS 4A is an ideal test site for models of magnetized cloud collapse and star formation. BIMA spectropolarimetric observations at $1.3 \mathrm{~mm}$ have detected and partially resolved the polarization in both the dust and $\mathrm{CO}$ (21) emission (Girart et al. 1999), showing hints of a hourglass morphology of the magnetic field. Recent polarimetric observations with the SMA at $877 \mu$ m with a resolution of 1'.3 (390 AU) have shown that the magnetic field associated with the infalling envelope has a clearly "pinched" morphology on a scale of a few hundreds AU (see Fig. 1 in Girart et al. 2006). This morphology resembles the hourglass shape that is predicted by the standard theory of low-mass star formation in a collapsing core with a regular magnetic field dominating the irregular (turbulent) one (Fiedler \& Mouschovias 1993; Galli \& Shu 1993a,b; Nakamura \& Li 2005). Applying the Chandrasekhar-Fermi equation, Girart et al. (2006) derived a magnetic field strength in the plane of the sky (POS) of $B_{\mathrm{POS}} \approx 5 \mathrm{mG}$, corresponding to a mass-to-flux ratio of $\sim 1.7$ times the critical value.

Gonçalves et al. (2008) compared the position angles in the plane of the sky of the polarization vectors determined by Girart et al. (2006) with the inclination of magnetic field lines of ideal (Galli \& Shu 1993a,b) and non-ideal (Shu et al. 2006) 
MHD collapse models. They found a good qualitative agreement for a source with $\lesssim 1 M_{\odot}$ and a mass-to-flux ratio of $\sim 2$ times the critical value. The present work is a step forward in the modelization and methodology with respect to that of Gonçalves et al. (2008).

This paper is organized as follows: in Sects. 2 and 3 we describe the target source IRAS 4A and the selected MHD models, respectively. In Sect. 4 we describe the synthetic map generation. General results are detailed in Sect. 5. In Sect. 6 we present the MHD models prediction convolved with the SMA interferometer and compare them with IRAS 4A observations. In Sect. 7 we present the ALMA maps of the MHD model prediction. Finally, in Sect. 8 we summarize the results and list the conclusions.

\section{NGC 1333 IRAS 4A}

The Perseus molecular cloud is an active low-mass star forming region, located at a distance ranging from $230 \mathrm{pc}$ to $350 \mathrm{pc}$ (Ridge et al. 2006). For this work, we adopt the value of $300 \mathrm{pc}$ (Girart et al. 2006). In the southern part of the reflection nebulae NGC 1333, Jennings et al. (1987) were the first to identify the protostar NGC 1333 IRAS 4. Sandell et al. (1991) resolved the system into two different components, IRAS 4A and IRAS 4B, separated by $\sim 31^{\prime \prime}$. They measured a luminosity of $\sim 28 L_{\odot}$ (at $350 \mathrm{pc}, 11 L_{\odot}$ at $220 \mathrm{pc}$ ) equally shared between the two components. Subsequent interferometric observations have revealed further multiplicity: IRAS 4A is itself a binary system. The two components IRAS 4A1 and IRAS 4A2 are separated by $540 \mathrm{AU}$ (1'.8, Lay et al. 1995; Looney et al. 2000; Girart et al. 2006).

This low-mass stellar system is in a very early stage of evolution. IRAS 4A and 4B are still embedded in a dense molecular and dusty envelope. Sandell et al. (1991) derived from submillimetric continuum single-dish observations a mass of $\sim 9 M_{\odot}$. Subsequent interferometric observations derived a mass of $1.2 M_{\odot}$ (Girart et al. 2006) for the compact component. Di Francesco et al. (2001) detected infall motions from inverse p-Cygni profiles observed in $\mathrm{H}_{2} \mathrm{CO}\left(3_{12}-2_{11}\right)$ and $\mathrm{N}_{2} \mathrm{H}^{+}(1-0)$. Single-dish CO (3-2) observations revealed a NE-SW wellcollimated outflow arising from IRAS 4A (Blake et al. 1995). Choi (2005) reports, through interferometric SiO (1-0) observations, a highly collimated NE-SW outflow with a projected position angle of $\sim 19^{\circ}$, and hints of a N-S outflow. The author proposes that IRAS 4A2 is powering the main outflow while IRAS 4A1 would power the secondary one.

\subsection{New data}

For this work we generated new observational maps of IRAS 4A combining compact (Girart et al. 2006), and sub-compact and extended (Ching \& Lai, priv. comm.) configuration SMA data (see left panel of Fig. 1), consisting of $8 \mathrm{~h}$ tracks in polarization mode at $880 \mu \mathrm{m}$. The data reduction was performed using MIRIAD, while the imaging was done using GREG from the GILDAS package. To obtain the maps we used a weighting robust parameter of 0.5 (Briggs 1995) corresponding to a beam of 1 '. $24 \times 1$ '. 12 , slightly smaller than that of Girart et al. (2006). Adding the sub-compact configuration improved the map with respect to that of Girart et al. (2006): the sampling of the larger scales is better and allows a better characterization of the circumbinary envelope. In addition, the extended configuration data help in separating the emission arising from either the embedded compact sources or the circumbinary envelope. The combined continuum (Stokes $I$ ) map is in good agreement with that of Girart et al. (2006), although the emission is more extended and has a sharper morphology. Furthermore, the polarized intensity map covers a larger area, has a slightly higher intensity peak and a more defined morphology.

The dust emission of IRAS 4A arises from the cold circumbinary envelope and from the warm circumstellar material around each protostar. Since the focus of this paper is on the morphology of the magnetic field in the circumbinary envelope, we have subtracted the contribution from the circumstellar component to the SMA visibility data. To do so, we first derived a map of the longest baselines (100-260 $k \lambda$ ) corresponding to a beam of $0.70 \times 00^{\prime} 46$. At these $u, v$-distances, the emission from the circumbinary envelope is resolved out, and the only contribution from the dust emissions arises from the circumstellar material (see central panel of Fig. 1 and Sect. 5.3). Then, the clean components of this map were subtracted from the original visibilities. Finally, we obtained a new map of the circumbinary envelope using the resulting visibilities (see right-hand side panel of Fig. 1).

Table 1 shows the main parameters of the emission associated only with the circumbinary envelope: peak position, $T_{\text {dust }}$, rms, $S_{v}, I_{v}^{\text {peak }}, F W H M, N_{\mathrm{H}_{2}}, n_{\mathrm{H}_{2}}$ and mass. The integrated flux is 4.1 Jy, corresponding to a mass of $0.8 M_{\odot}$, both slightly smaller than those of Girart et al. (2006) as we were able to isolate the envelope. The optical depth of the dust emission at $880 \mu \mathrm{m}$ imply that the observations trace very deep into the source. Therefore, neglecting scattering (see Sect. 4.1) and assuming an anisotropic radiation field, the polarized dust emission is probably originated in the alignment of dust grains to the magnetic field (see Lazarian 2003, for a comprehensive review on this topic). The envelope hourglass morphology of the magnetic field is more evident than in earlier data. A new feature is the double peak in polarized intensity. The map also shows a significant depolarization toward the source main axis, which was not as clear in the Girart et al. (2006) map. This feature can be explained in terms of projections effects intensified by beam smearing (see, e.g., Gonçalves et al. 2005).

\section{Theoretical models}

We compare the dust polarization map of IRAS 4A described in the previous section with the predictions of three models of magnetized cloud collapse. The models of Galli \& Shu (1993a,b) and Allen et al. (2003a,b) give the density profile and magnetic field distribution of an infalling envelope surrounding a low-mass star, the two models differing mainly in the choice of the initial conditions. The Shu et al. (2006) model is similar to the previous two, but contains a parameter representing the spatial scale where the diffusive effects associated to an electric resistivity (assumed uniform) dominate the evolution of the magnetic field. Therefore, our analysis is not able to test the theory of core formation from iniatially subcritical conditions by ambipolar diffusion. This can only be accomplished by spatially resolved Zeeman observations of molecular cloud cores and their surroundings (see e.g., Crutcher et al. 2009). In a following paper (Frau et al., in prep.) we will analyze synthetic polarization maps of protostellar cores extracted from numerical simulations of turbulent clouds.

\subsection{Galli \& Shu $(1993 a, b)$}

This model follows the collapse of a singular isothermal sphere threaded by an initially uniform magnetic field. The cloud is assumed to be non rotating. This initial condition is a highly idealized representation of a non-equilibrium state. Inside the 
P. Frau et al.: Comparing star formation models with interferometric observations. I.

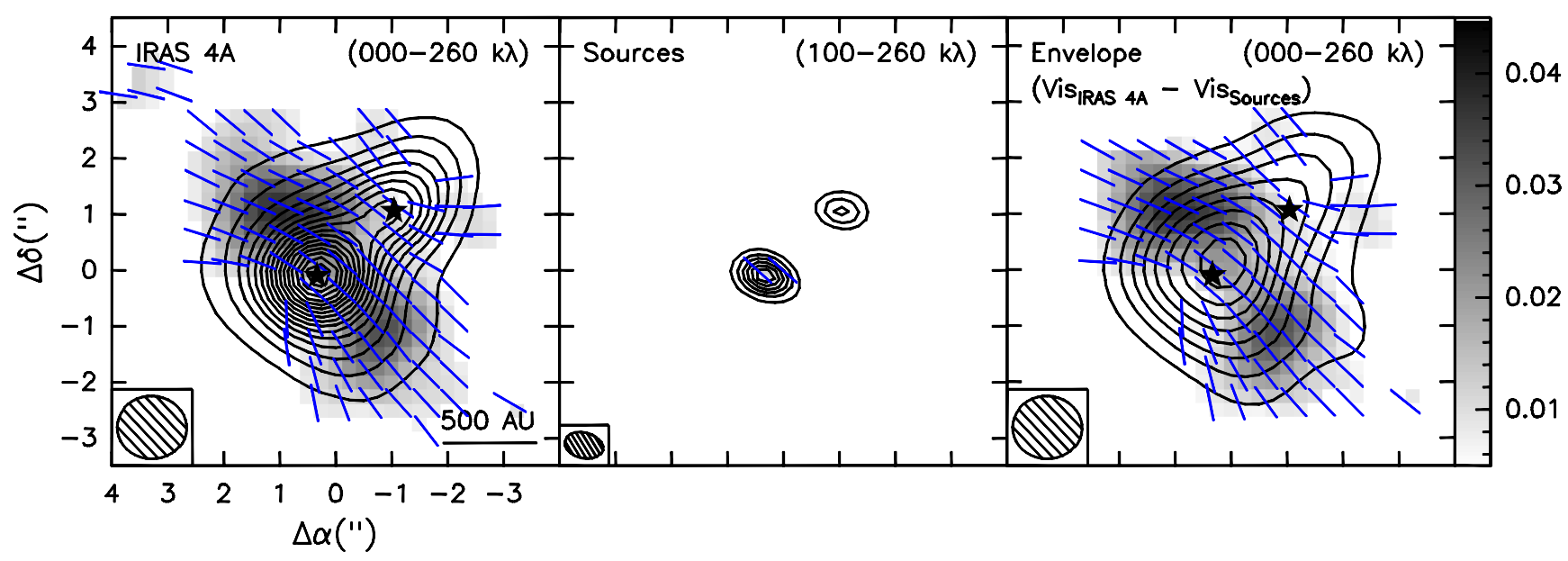

Fig. 1. Left panel: IRAS 4A combining SMA sub-compact, compact and extended configurations with robust parameter set to 0.5. The stars mark the two compact sources (see central panel). Top-right corner shows the $u, v$ range used. The synthesized beam is $11^{\prime \prime} 24 \times 1$ "' 12 . Contours show the dust emission at $880 \mu \mathrm{m}$ in steps of $6 \sigma$, from $6 \sigma$ to $96 \sigma$, where $\sigma=0.02 \mathrm{Jy} \mathrm{beam}^{-1}$. The pixel map shows the polarized intensity (see scale on the right-hand side). Blue bars show the observed magnetic field direction. Central panel: IRAS 4A compact components. Legends are the same as in

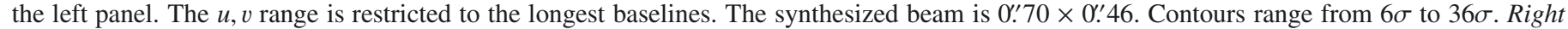
panel: IRAS 4A envelope emission (see Sect. 2.1). Legends are the same as in the left panel. Contours range from $6 \sigma$ to $48 \sigma$.

Table 1. IRAS 4A: envelope continuum emission at $880 \mu \mathrm{m}$ and derived parameters $^{a}$.

\begin{tabular}{|c|c|}
\hline $\begin{array}{l}\alpha(\mathrm{J} 2000)^{b} \\
\delta(\mathrm{J} 2000)^{b} \\
T_{\text {dust }}{ }^{c} \\
\end{array}$ & $\begin{array}{c}3^{\mathrm{h}} 29^{\mathrm{m}} 10.520^{\mathrm{s}} \\
31^{\circ} 13^{\prime} 31^{\prime \prime} .12 \\
50 \mathrm{~K} \\
\end{array}$ \\
\hline $\begin{array}{l}\mathrm{rms}_{\mathrm{I}} \\
\mathrm{rms}_{\mathrm{Q}} \\
\mathrm{rms}_{\mathrm{U}}\end{array}$ & $\begin{array}{l}20 \mathrm{mJy} \mathrm{beam}^{-1} \\
2.5 \mathrm{mJy} \mathrm{beam}^{-1} \\
2.5 \mathrm{mJy} \mathrm{beam}^{-1}\end{array}$ \\
\hline $\begin{array}{l}S_{v}^{\mathrm{I}} \\
I_{\text {peak }}^{\mathrm{I}} \\
F W H M^{d}\end{array}$ & $\begin{array}{c}4.1 \pm 0.4 \mathrm{Jy} \\
1.03 \pm 0.02 \mathrm{Jy} \mathrm{beam}^{-1} \\
1156 \mathrm{AU}\left(3^{\prime \prime} .85\right)\end{array}$ \\
\hline $\begin{array}{l}S_{v}^{\mathrm{pol}} \\
I_{\text {peak }}^{\text {pol }} \\
\Omega^{\text {pole }} \\
\end{array}$ & $\begin{array}{c}160 \pm 16 \mathrm{mJy} \\
38.2 \pm 2.5 \mathrm{mJy} \mathrm{beam}^{-1} \\
14 \mathrm{arcsec}^{2}\end{array}$ \\
\hline $\begin{array}{l}\tau \\
N_{\mathrm{H}_{2}}{ }^{f} \\
n_{\mathrm{H}_{2}}{ }^{f} \\
\text { Mass }^{f}\end{array}$ & $\begin{array}{c}0.07 \\
1.2 \times 10^{24} \mathrm{~cm}^{-2} \\
1.1 \times 10^{8} \mathrm{~cm}^{-3} \\
0.8 M_{\odot}\end{array}$ \\
\hline
\end{tabular}

Notes. ${ }^{(a)}$ See Appendix A of Frau et al. (2010) for details. ${ }^{(b)}$ From a 2D Gaussian fit to the source. ${ }^{(c)}$ Girart et al. (2006). ${ }^{(d)}$ Diameter of the circle with the same area as the region of the source with intensity above half of the peak. ${ }^{(e)}$ Solid angle of the region with polarized intensity above $3 \sigma .{ }^{(f)}$ Assuming $\kappa_{250 \mathrm{GHz}}=1.5 \mathrm{~cm}^{2} \mathrm{~g}^{-1}$ and a gas-to-dust ratio of 100 (Girart et al. 2006).

collapse region, bounded by an outward propagating slow magnetosonic wave, the magnetic field dragged by the flow (even in the presence of ambipolar diffusion) deflects the infalling gas towards the midplane, forming a large pseudodisk. The initial state depends on two dimensional quantities, $r_{0}=2 a^{2} / \sqrt{G} B_{0}$ and $t_{0}=2 a / \sqrt{G} B_{0}$, defining the characteristic spatial and temporal scale of the collapse. These depend on the sound speed $a$, the gravitational constant $G$ and the initial (uniform) magnetic strength $B_{0}$. For given $r_{0}$ and $t_{0}$, the time evolution depends on the non-dimensional parameter $\tau=t / t_{0}$, where $t$ is the time elapsed since the onset of collapse. Fixing $a=0.35 \mathrm{~km} \mathrm{~s}^{-1}$, the model thus depends only on $B_{0}$ and $\tau$.

\subsection{Allen et al. $(2003 a, b)$}

This model is similar to that of Galli \& Shu (1993a,b) with some important differences: $(i)$ being fully numerical, it overcomes the spatial and temporal limitations of the semi-analytical approach of Galli \& Shu (1993a,b); (ii) the initial state is a magnetostatic unstable equilibrium configuration (a "singular isothermal toroid" see Li \& Shu 1996), already flattened in the direction perpendicular to a magnetic field possessing a hourglass morphology from the start; (iii) the cloud can rotate around an axis parallel to the axis of the magnetic field. As in the Galli \& Shu (1993a,b), magnetic field lines internal to a "separatrix" are dragged into the accreting protostar.

The initial configuration is specified by the sound speed, $a$ (as in the Galli \& Shu 1993a,b model), and the level of magnetic to thermal support, $H_{0}$, which represents the fractional overdensity supported by the magnetic field above that supported by the thermal pressure, and the rotational speed, $v_{0}$. The parameter $H_{0}$ is related to the mass-to-flux ratio of the cloud.

The flattening of the mass distribution (the "pseudodisk") and the magnetic field geometry are little affected by rotation. Conversely, the angular velocity of the infalling gas is strongly influenced by the magnetic braking associated to the strong field created by accretion, assuming ideal MHD. This effect has important implications for the formation of rotationally supported disks around young stars (see Galli et al. 2006).

\subsection{Shu et al. (2006)}

To overcome the difficulties associated to catastrophic magnetic braking and to the huge magnetic flux of the protostar, Shu et al. (2006) consider the consequences of non-ideal MHD effects during the accretion phase of low-mass star formation. In steady state, magnetic dissipation occurs inside a region of radius equal to the so-called "Ohm radius", $r_{\mathrm{Ohm}}=\eta^{2} /\left(2 G M_{\star}\right)$, where $\eta$ is the Ohmic resistivity (assumed uniform), $G$ is the gravitational constant, and $M_{\star}$ is the mass of the accreting protostar. Outside $r_{\mathrm{Ohm}}$, the accreting gas is in free fall along radial field lines, that become straight and uniform inside $r_{\mathrm{Ohm}}$. The magnetic flux accreted by the central protostar is zero at all times. 


\section{Synthetic map generation}

\subsection{Assumptions}

To compare the intensity and the polarized intensity predicted by the models with the observed data, it is important to consider the effects of a temperature gradient, since the sub-mm emission is roughly proportional to the temperature. In this work, we have assumed both a uniform temperature profile (UTP) and a radial temperature profile (RTP) derived for IRAS 4A by Maret et al. (2002) from water emission. Although the theoretical models considered here are computed assuming an isothermal equation of state, the IRAS 4A observed temperature gradient does not significantly affect the dynamics of collapse, because the kinetic energy due to thermal motions is more than one order of magnitude smaller than the kinetic energy of the infalling particles. For example, the temperature expected at $600 \mathrm{AU}$ is $50 \mathrm{~K}$, which leads to a thermal broadening of $\sigma_{\text {therm }} \sim 0.4 \mathrm{~km} \mathrm{~s}^{-1}$, whereas the infall velocity expected is $v_{\mathrm{ff}} \sim 1.7 \mathrm{~km} \mathrm{~s}^{-1}$.

We consider optically thin emission with no absorption or scattering effects, in agreement with the sub-mm emission properties (Hildebrand 1983; Novak et al. 1989) and with the opacity derived in IRAS 4A (see Table 1). We have assumed uniform grain properties, represented by the polarizing efficiency parameter $\alpha$ which includes the absorption cross section and the alignment efficiency. Following Fiege \& Pudritz (2000), the maximum degree of polarization is

$p_{\max }=\frac{\alpha}{1-\alpha / 6}$.

We assumed $p_{\max }=15 \%$, corresponding to $\alpha=0.15$. Despite the high value of $\alpha$ used, and the fact that the grain properties may change with density (Fiege \& Pudritz 2000), we find that the absolute polarized intensities derived in the models match reasonably well the observed values in IRAS 4A. Lower values of $\alpha$ (e.g., $\alpha=0.1$ ) did not reproduce the data equally well.

We performed the numerical integration using an equally spaced regular cubic grid and uniform step in the line-of-sight direction.

\subsection{Method}

We improved the technique developed by Gonçalves et al. (2008) to compare theoretical models with observed data, including in the process the instrumental effects. In practice, we simulated all the steps of a regular observing run with both SMA and ALMA, generating synthetic maps with the same filtering and processing as the observed maps. With this technique we avoid any possible misinterpretation due to the effects of the instrumental response and filtering, as well as the data modification because of the Fourier transform of the observed visibilities and the subsequent application of the dirty map cleaning algorithm. The process consists of a series of 5 consecutive steps for each realization:

1. For any given model we generated three-dimensional (3D) data cubes of density and magnetic field components. The orientation in space of the 3D source models were defined by two viewing angles: the position angle $\phi$ of the projection of the polar axis in the plane of the sky with respect to the north direction, and the inclination angle $\omega$ of the polar axis with respect to the plane of the sky $\left(\omega=0^{\circ}\right.$ for edge-on view). Since the models used have axial symmetry, the optically thin emission assumption allowed us to explore only half of the inclination angle space $\left(0^{\circ} \leq \omega \leq 90^{\circ}\right)$. We restricted $\phi$ to the range $0^{\circ} \leq \phi \leq 90^{\circ}$ (the observations fix the magnetic axis of IRAS 4 A at $\phi \approx 50^{\circ}$, see Girart et al. 2006).

2. In the plane of the sky we simulated a square area with side length of $51^{\prime \prime} .2\left(\sim 1.5 \times 10^{4} \mathrm{AU}\right.$ at the distance of IRAS 4A). The map size was chosen to be about twice the SMA primary beam to better process the sidelobes in the final maps. In this plane we used a grid of $512 \times 512$ pixels with a pixel size of 0.1 ( $~ 30 \mathrm{AU})$, enough to oversample the smallest beam used in this work $\left(\sim 0.4^{\prime \prime} \sim 120\right.$ AU for ALMA). With this choice we ensured, in the final convolved maps, the independence of points separated more than a beam distance due to beam convolution. In the line-of-sight direction we covered a length of $6 \times 10^{3} \mathrm{AU}$ (equivalent to $20^{\prime \prime}$ ) sampled with 60 cells. A larger integration length or a larger number of steps did not affect significantly the details of the final maps.

3. Through a ray-tracing scheme, we integrated the emission of the cells along the line-of-sight $\ell$ generating $2 \mathrm{D}$ raw synthetic maps for the Stokes parameter $I, Q$, and $U$. We followed a method developed by Lee \& Draine (1985), and elaborated by Wardle \& Konigl (1990), Fiege \& Pudritz (2000), and Padoan et al. (2001). One can calculate the Stokes $Q$ and $U$ intensities as $Q=C q$ and $U=C u$, where $C$ is a constant that includes all the terms assumed to be constant (polarization efficiency and polarization and absorption cross sections) that can be interpreted as a polarized intensity scale factor. $q$ and $u$ are the "reduced" Stokes parameters defined as

$q=\int \rho B_{\lambda}\left(T_{\mathrm{d}}\right) \cos 2 \psi \cos ^{2} \gamma \mathrm{d} \ell$,
$u=\int \rho B_{\lambda}\left(T_{\mathrm{d}}\right) \sin 2 \psi \cos ^{2} \gamma \mathrm{d} \ell$,

where $\rho$ is the density, $B_{\lambda}\left(T_{\mathrm{d}}\right)$ is the Planck function at the dust temperature $T_{\mathrm{d}}, \psi$ is the angle between the north direction in the plane of the sky and the component of $\boldsymbol{B}$ in that plane, and $\gamma$ is the angle between the local magnetic field and the plane of the sky. Stokes $I$ is given by $I=(C / \alpha)\left(\Sigma-\alpha \Sigma_{2}\right)$ (Fiege \& Pudritz 2000) where $\alpha$ is the maximum polarizing efficiency assumed to be $15 \%$, the $C / \alpha$ factor can be interpreted as a total intensity scale factor, while $\Sigma$ and $\Sigma_{2}$ are defined as

$$
\begin{aligned}
\Sigma & =\int \rho B_{\lambda}\left(T_{\mathrm{d}}\right) \mathrm{d} \ell, \\
\Sigma_{2} & =\int \rho B_{\lambda}\left(T_{\mathrm{d}}\right)\left(\frac{\cos ^{2} \gamma}{2}-\frac{1}{3}\right) \mathrm{d} \ell,
\end{aligned}
$$

and represent the emitted Stokes $I$ intensity and the polarization absorption losses respectively. As for Stokes $Q$ and $U$, one can define the "reduced" Stokes I parameter as

$i=\frac{\Sigma-\alpha \Sigma_{2}}{\alpha}$

4. Once the synthetic Stokes 2D maps were generated, the flux was rescaled so that the total flux in the region of the map with $I_{v}>6 \sigma$ (see Sect. 2.1) matched that of the synthetic map. A Gaussian noise was added to match the noise of the observations for each Stokes map. These maps were converted to visibilities using the same visibility coverage in the $u, v$ plane as the real observations. In this step we mimicked the effects of the observation noise and the instrumental filtering. This allowed us to be sensitive to the same spatial 
P. Frau et al.: Comparing star formation models with interferometric observations. I.

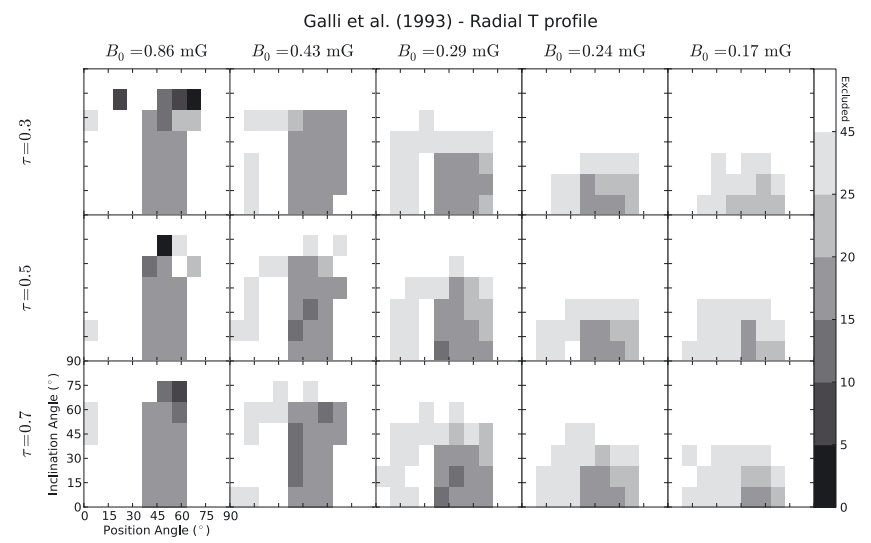

Fig. 2. Pixel map: standard deviation of the difference of the synthetic polarization vectors with respect to the observed ones for the Galli \& Shu (1993a,b) model with radial temperature profile. White pixels represent excluded models (see text). Grayscale is on the right-hand side of the figure.

scales and to similar emission levels. Then, the final model maps for the Stokes $I, Q$ and $U$ were obtained from the synthetic visibilities in the same way as the combined SMA maps presented in Fig. 1.

5. The process described in points 1 to 4 is repeated a large number of times for both temperature treatments using different values for $(i)$ the position angle $\phi$ and the inclination angle $\omega$, and (ii) the model parameters.

For ALMA, we used the task simdata from the CASA package to predict the expected maps for the models with the ALMA capabilities. At the source distance, the cells of the simulations had a typical length of $\sim 30$ AU. We chose the full ALMA configuration 09 , which provides a synthesized beam of $0.7 \times 0 . ' 4$ $(210 \times 120 \mathrm{AU})$ thus ensuring that the final synthetic maps were not affected by resolution issues. We simulated a $2 \mathrm{~h}$ run at $345.8 \mathrm{GHz}$ in polarization mode. As good weather is required for polarization measurements, we assumed $1 \mathrm{~mm}$ of precipitable water vapor. The elevation of the source ranged between $30^{\circ}$ and $40^{\circ}$.

\subsection{Selection}

At this point, one has to select the "best" procedure for comparing the synthetic maps with the observational data. This can be accomplished in several ways: (1) using the method of Gonçalves et al. (2008) based on the minimization of the difference between the observed and predicted position angles of the polarization vectors (hereafter simply "angle difference method"); (2) performing a $\chi^{2}$ analysis of the synthetic Stokes $Q$ and $U$ maps with respect to the observed maps. In the latter case (hereafter simply " $\chi 2$ method"), we positioned the peak of the synthetic map on the peak of the IRAS 4A envelope (see Fig. 1), and we compared the synthetic map with all the region of the observed map with intensity larger than $3 \sigma$ (see Table 1). As we focus on the polarized emission, we define the best fitting models as those which minimize the sum $\chi^{2}=\chi_{Q}^{2}+\chi_{U}^{2}$. Stokes $I$ was excluded since it shows considerable dependence on the assumed temperature profile.

We illustrate the results for the two selection methods for the Galli \& Shu (1993a,b) models with RTP (see Figs. 2 and 3). Figure 2 shows the standard deviation of the distribution of differences in position angles as function of the viewing angles $\omega$

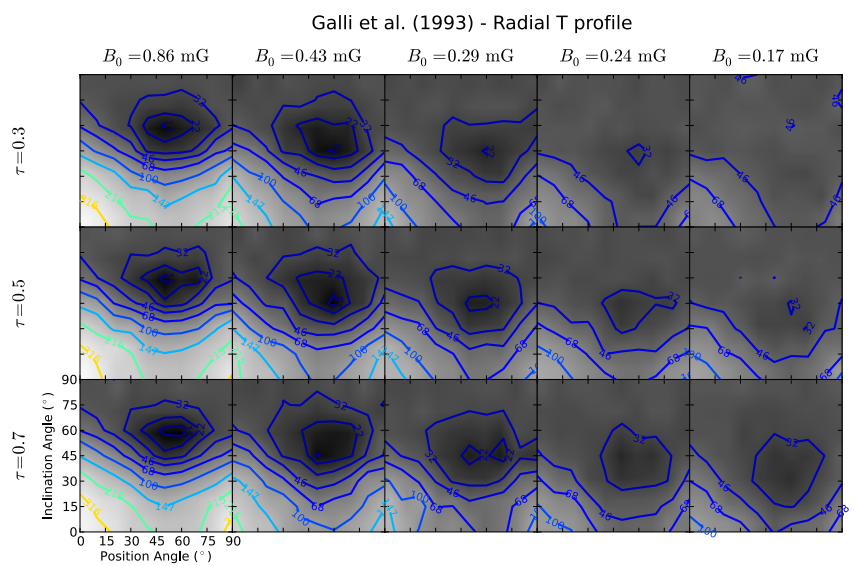

Fig. 3. Pixel map: sum of the $\chi^{2}$ of the Stokes $Q$ and $U$ maps for the Galli \& Shu $(1993 a, b)$ model with radial temperature profile with respect to the data. Contours: $10,10^{1 / 6}, 10^{2 / 6}, \ldots, 10^{3} \chi^{2}$ levels.

and $\phi$ for the Galli \& Shu (1993a,b) models described before. Only runs with average difference value of the position angles with respect to those of IRAS 4A lower than $15^{\circ}$ are shown. Best-fitting models are characterized by the smallest values of the standard deviation. The uniform distribution of results makes evident the low discrimination power of the angle difference method. Conversely, the $\chi^{2}$ method allows to perform a more significant selection of the best-fitting models. Figure 3 shows a difference of more than one order of magnitude between bad- and well-fitting runs thus providing a higher discriminating power among all the runs. Therefore, in the rest of this paper, we adopt the $\chi^{2}$ selection method.

\section{Results}

\subsection{Orientation angles}

Stokes $Q$ and $U$ maps show a significant dependence on the orientation angles of the source, and can be used to constrain the viewing geometry. Figure 4 illustrates the different emission patterns arising from an Allen et al. (2003a,b) source, with $H_{0}=0.125, v_{0}=0$, and $t=2 \times 10^{4} \mathrm{yr}$, after varying the orientation angles. Stokes $Q$ and $U$ maps are shown for all the combinations of position $(\phi)$ and inclination $(\omega)$ angles of $0^{\circ}, 30^{\circ}, 45^{\circ}$, $60^{\circ}$, and $90^{\circ}$. Conversely, Fig. 5 shows Stokes $I$ maps which depend marginally on the position angle, and only for small inclination angles.

As can be seen in Fig. 4, Stokes $Q$ is specially sensitive to the position angle for small inclination angles, while Stokes $U$ appear to vary more with inclination angle at non-extreme position angles. From a practical point of view, one could identify with relatively high precision, by pure comparison with Figs. 4 and 5, both position angles of a source with a magnetic field with hourglass morphology and an inclination angle smaller than $\sim 60^{\circ}$. For inclination angles $\gtrsim 60^{\circ}$ the expected magnetic field tend to be mostly radial and the Stokes $Q$ and $U$ maps show very similar morphologies independently of $\omega$. Therefore, polarization observations have shown to be a powerful tool to determine both position angles of a sources with respect to the LOS direction. Figures 4 and 5 could be used as templates for future observations of the dust polarized emission toward star forming cores. 
A\&A 535, A44 (2011)

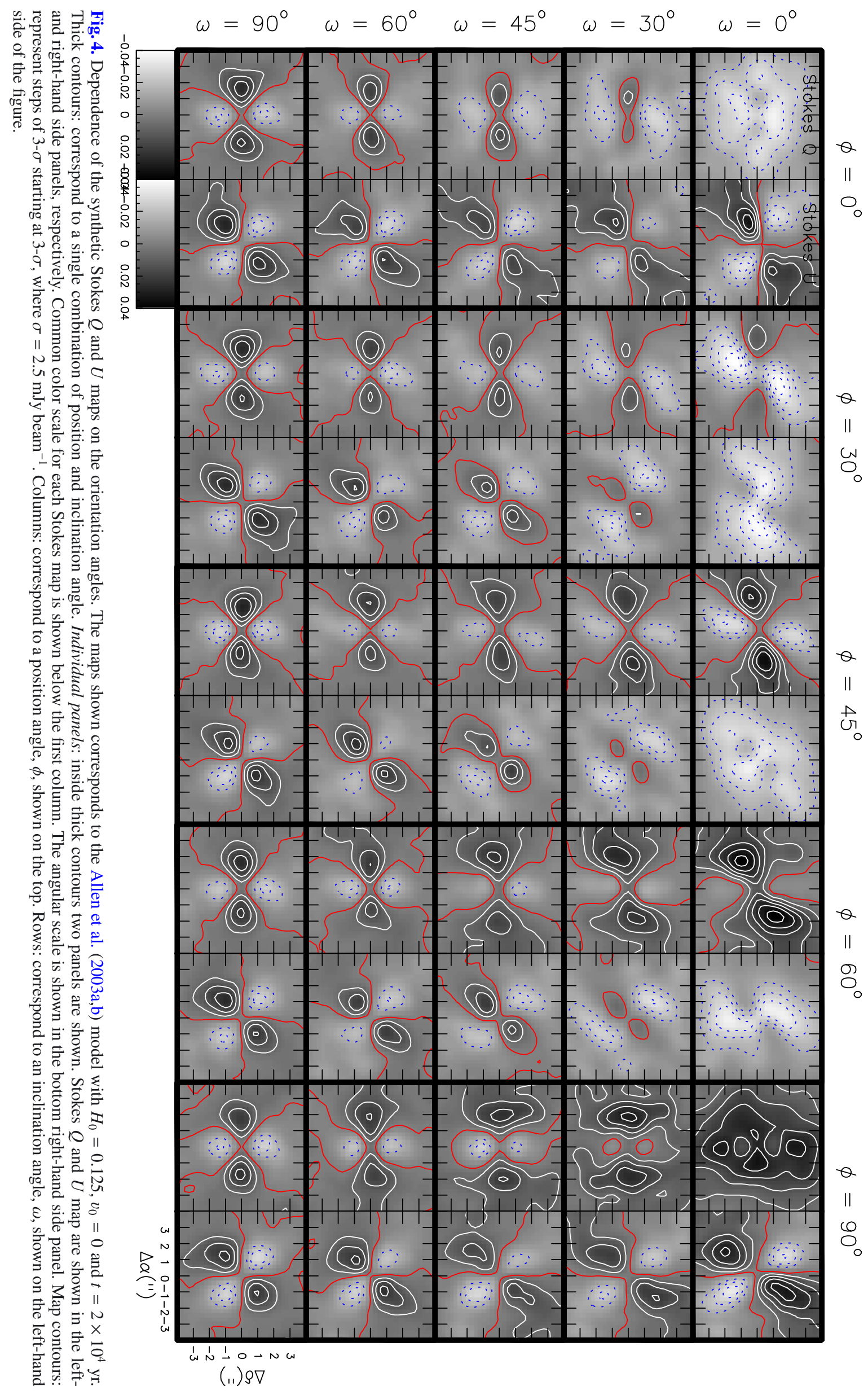


P. Frau et al.: Comparing star formation models with interferometric observations. I.

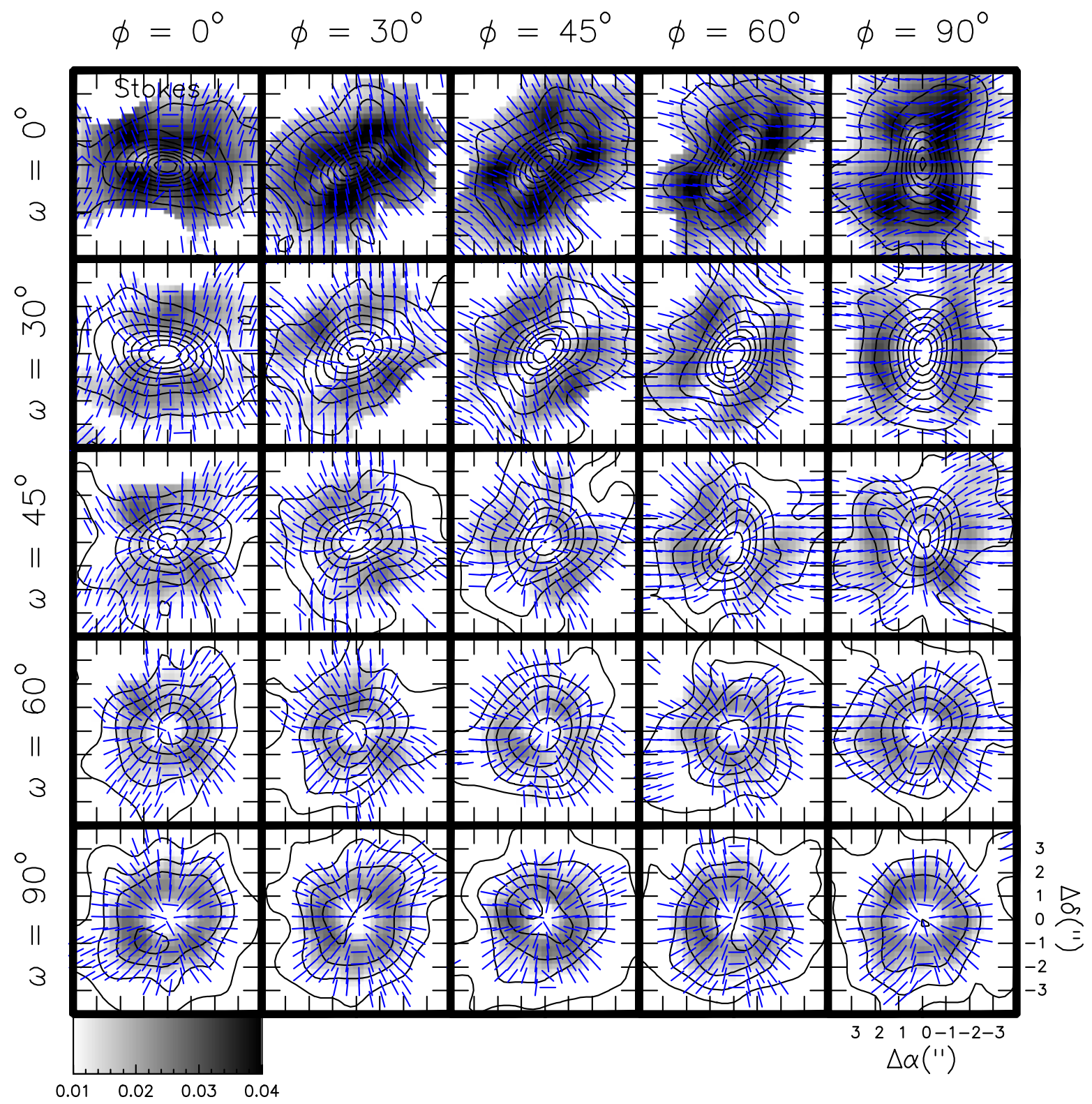

Fig. 5. Same as Fig. 4 but for Stokes $I$ maps. Each panel show the total emission (contours), the polarized emission (pixel map) and the magnetic field direction (segments). Map contours: for Stokes $I$ represent steps of $6-\sigma$ starting at $6-\sigma$, where $\sigma=0.02 \mathrm{Jy} \mathrm{beam}^{-1}$.

\subsection{Temperature profiles}

Figure 6 shows the differences in Stokes $I u, v$ amplitude between both temperature treatments. The models show more realistic amplitudes using the RTP. The models with UTP show lower intensities than IRAS 4 A in the $\gtrsim 25 k \lambda$ range $\left(\$ 4^{\prime \prime}\right)$, while the models with RTP fit the observations up to $15-20 k \lambda\left(\lesssim 5^{\prime \prime} .5\right)$. Note that this is remarkable for either temperature treatment as the intensity rescaling was done in the $I_{v}>6 \sigma$ which covered radii $\lesssim 3^{\prime \prime}(\gtrsim 35 k \lambda)$. Figures 7 and 8 show the same information in the image domain for UTP and observed RTP, respectively, for cases with realistic model parameters and orientation angles. UTP maps show more extended emission (larger $u, v$ amplitudes at short baselines) than IRAS 4A and a lower emission peak evident from the residual maps. On the other hand, RTP maps show a slightly more extended morphology than IRAS 4A and realistic intensity peak values. Note that RTP residual maps tend to show zero emission at short radii and slightly negative emission at large ones due to the more extended sources predicted by the models. More realistic fluxes, intensity peaks and masses are derived from the RTP treatment. However, even using the observed temperatures, the radial intensity profile from models is steeper than that of IRAS 4A, which lead to smaller FWHM for model synthetic maps and, consequently, higher densities than those observed.

The RTP treatment also predicted more realistic Stokes $Q$ and $U$ maps, shown in the middle left-hand side and middle right-hand side panels of Figs. 7 and 8. Although peak values are similar for both treatments, RTP map show roughly the same polarized flux over the same solid angle with similar morphology to IRAS 4A. On the other hand, UTP map showed roughly twice as much polarized flux over twice the solid angle of IRAS 4A (see Tables 2 and 3). An immediate consequence was the unrealistic UTP vector map whereas the RTP one reasonably match that of IRAS 4A.

Summarizing, RTP maps reproduced with higher fidelity the observed IRAS 4A emission, in the three Stokes parameters, better than UTP maps in the same conditions. Consequently, the physical parameters derived from RTP maps were more realistic. 


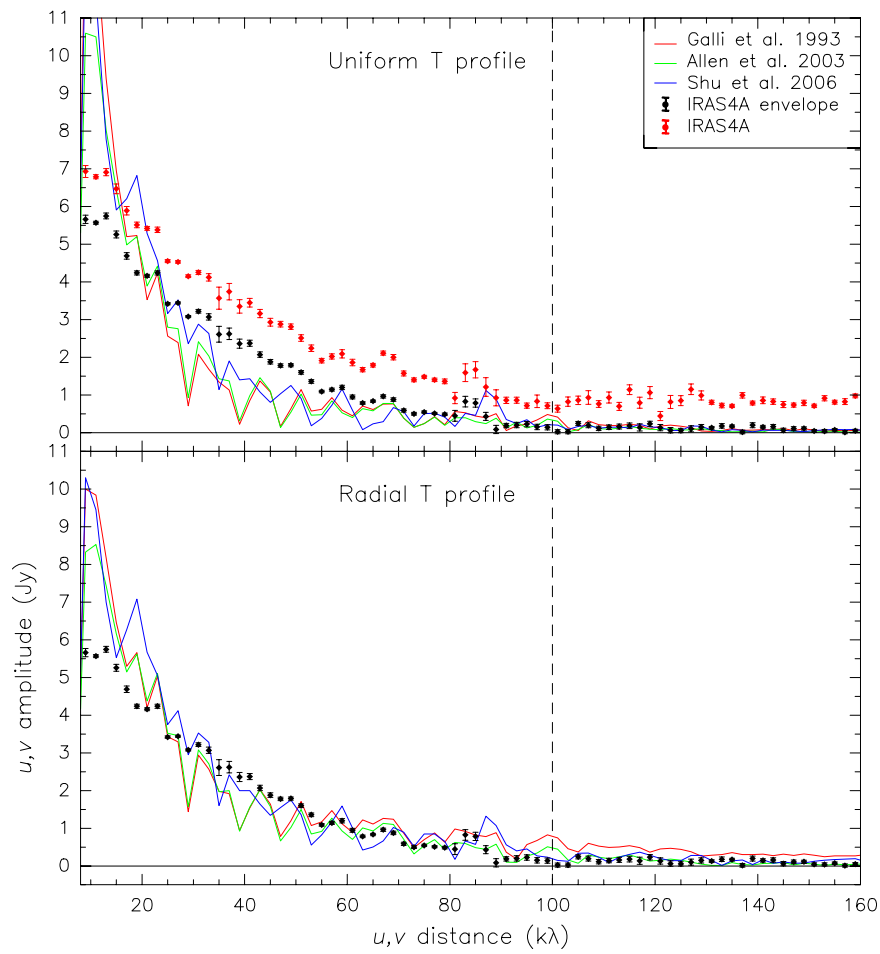

Fig. 6. Stokes $I$ visibility amplitudes vs. $u, v$ distance averaged in bins of $2 k \lambda$. Top panel: uniform temperature profile. Bottom panel: radial temperature profile. Red and black dots: IRAS 4A full data and envelope data, respectively, with their statistical error bars. Vertical dashed line: $u, v$ distance threshold used to derive the compact components map (see Fig. 1). Starting at $\sim 90 k \lambda$ the emission seems to match with an unresolved source of $\sim 1$ Jy. Red, green, and blue solid curves: visibility amplitudes derived after convolving the Galli \& Shu (1993a,b), Allen et al. (2003a,b), and Shu et al. (2006) models, respectively, used to generate the maps of left-hand panels of Figs. 7 and 8.

\subsection{Visibility amplitudes}

Solid curves in Fig. 6 show the total $u, v$ amplitude as a function of the $u, v$ distance for the models using realistic model parameters and orientation angles $\left(\phi=50^{\circ}\right.$ and $\omega=45^{\circ}$, see Sects. 5.1 and 6). The oscillations in the synthetic visibilities are due to the added noise. The intensity rescaling of synthetic data was performed in the image domain as it is the output of the simulations. However, a good agreement in the $u, v$ data is important given that it is the output from the telescope. Red and black dots in Fig. 6 show, respectively, the resulting $u, v$ amplitudes before and after subtracting the two circumstellar compact components. We assumed that only emission from the envelope remained after subtraction. To test the goodness of the rescaling method we compared the observed $u, v$ data of IRAS 4A with the synthetic $u, v$ data derived from the models. A remarkable agreement (specially using the observed temperature profile, see Sect. 5.2) is achieved for $u, v$ distances ranging from $\sim 20 k \lambda$ up to the maximum baseline with significant envelope emission $(\sim 90 \mathrm{k} \lambda)$. None of the envelope models showed significant emission at $u, v$ distances $\gtrsim 100 k \lambda$ (equivalent to a radius of $1^{\prime \prime}$ or $300 \mathrm{AU})$ reinforcing the hypothesis that no envelope emission is detected from the IRAS 4A envelope in this $u, v$ range. At $u, v$ distances shorter than $\sim 20 k \lambda$ the models show larger emission than the IRAS 4A envelope. At a distance of $300 \mathrm{pc}$ this scale is equivalent to emission with a radius of $\gtrsim 1500 \mathrm{AU}\left(25^{\prime \prime}\right)$, which is larger than the radius of IRAS $4 \mathrm{~A}$ at a $3-\sigma$ level $\left(\sim 3^{\prime \prime}\right.$, $\sim 900 \mathrm{AU})$. This excess has its origin in the fact that the models predict a more extended source than the observed one, with typical radius of $\sim 4^{\prime \prime}(\sim 1200 \mathrm{AU})$ at a 3- $\sigma$ level.

\section{SMA synthetic maps}

In general, synthetic intensity maps obtained from theoretical models tend to be less concentrated than the observed sources. The flux scaling based on the real data, combined with less compact synthetic sources, cause the synthetic maps to show more extended emission than the observed ones, and also a lower flux intensity peak. In the following subsections $(6.1,6.2$, and 6.3) we determine the best fitting parameters for each individual model (see Figs. 3, 9, and 10) and perform a direct comparison of IRAS 4A with all the models (see Figs. 7 and 8, and Tables 2 and 3).

\subsection{Galli \& Shu $(1993 a, b)$}

We selected 5 values of the initial magnetic field $\left(B_{0}=0.86 \mathrm{mG}\right.$, $0.43 \mathrm{mG}, 0.29 \mathrm{mG}, 0.24 \mathrm{mG}$, and $0.17 \mathrm{mG}$, corresponding to $t_{0}=10^{4} \mathrm{yr}, 2 \times 10^{4} \mathrm{yr}, 3 \times 10^{4} \mathrm{yr}, 4 \times 10^{4} \mathrm{yr}$, and $\left.5 \times 10^{4} \mathrm{yr}\right)$ and 3 values of the non-dimensional time $\tau(\tau=0.3,0.5$ and 0.7$)$. The mass-to-flux ratio of the initial configuration is not spatially uniform as in the models of Allen et al. (2003a,b) described in Sect. 5.3. A spherical region centered on the origin and enclosing a mass $M$ has a mass-to-flux ratio $M / \phi=\pi c_{\mathrm{s}}^{2} /\left(B_{0} G^{2} M\right)$. With the values of $B_{0}$ listed above, and for a region enclosing a mass $M=1 M_{\odot}$, this corresponds to a mass-to-flux ratio, in units of the critical value, of 1.0, 2.0, 3.0, 3.6 and 5.1.

For each choice of $B_{0}$ and $\tau$ we ran 70 cases corresponding to 10 different values of the position angle $\phi$ and 7 values of the inclination angle $\omega$. We considered both an isothermal source and a radial temperature profile (see Sect. 4.1). For each of the 2100 maps generated, the model Stokes $Q$ and $U$ were compared with the observed values, and the sum of individual $\chi^{2}$ was evaluated (see the radial temperature profile results in Fig. 3). The results show that the best fit to the data is given by the models with the highest values of the initial magnetic field $B_{0}$. In all cases, intermediate values of $\phi$ and $\omega$ are selected. Note also that for smaller values of $B_{0}$, the best fit is achieved for lower values of $\omega$. This is due to a zooming effect: a larger $B_{0}$ implies a smaller $r_{0}$. For large $B_{0}$ smaller angular distances mean larger radii, where the magnetic field configuration of the outermost parts of the model are naturally pinched in an edge-on view. On the other hand, for small $B_{0}$ (large $r_{0}$ ), the innermost region the magnetic field tend to be radial, and a larger inclination angle combined with the line-of-sight emission integration is needed to produce the pinched morphology.

The fit is not sensitive to the value of the non-dimensional time $\tau$. Thus, the time elapsed since the onset of collapse is not well constrained by these models. For example, for models with $B_{0}=0.29 \mathrm{mG}$ (third column in Fig. 3) the time corresponding to the three values of $\tau$ is $8.7 \times 10^{3} \mathrm{yr}, 1.5 \times 10^{4} \mathrm{yr}$, and $2.0 \times 10^{4} \mathrm{yr}$, whereas for models with $B_{0}=0.17 \mathrm{mG}$ (fifth column), the time range corresponding to the three values of $\tau$ is $1.5-3.5 \times 10^{4} \mathrm{yr}$.

Figures 7 and 8 show the predicted Stokes $I, Q, U$ maps and the Stokes $I$ residuals (second row) compared to the observed maps (first row) for this model, for the case with uniform temperature and temperature gradient, respectively. Both realizations shown have $B_{0}=0.43 \mathrm{mG}, \tau=0.7$ (corresponding to $t=1.4 \times 10^{4} \mathrm{yr}$ after the onset of collapse), $\phi=50^{\circ}$ and $\omega=45^{\circ}$. The derived physical parameters are shown in Tables 2 and 3 for the case with uniform temperature and temperature 
P. Frau et al.: Comparing star formation models with interferometric observations. I.

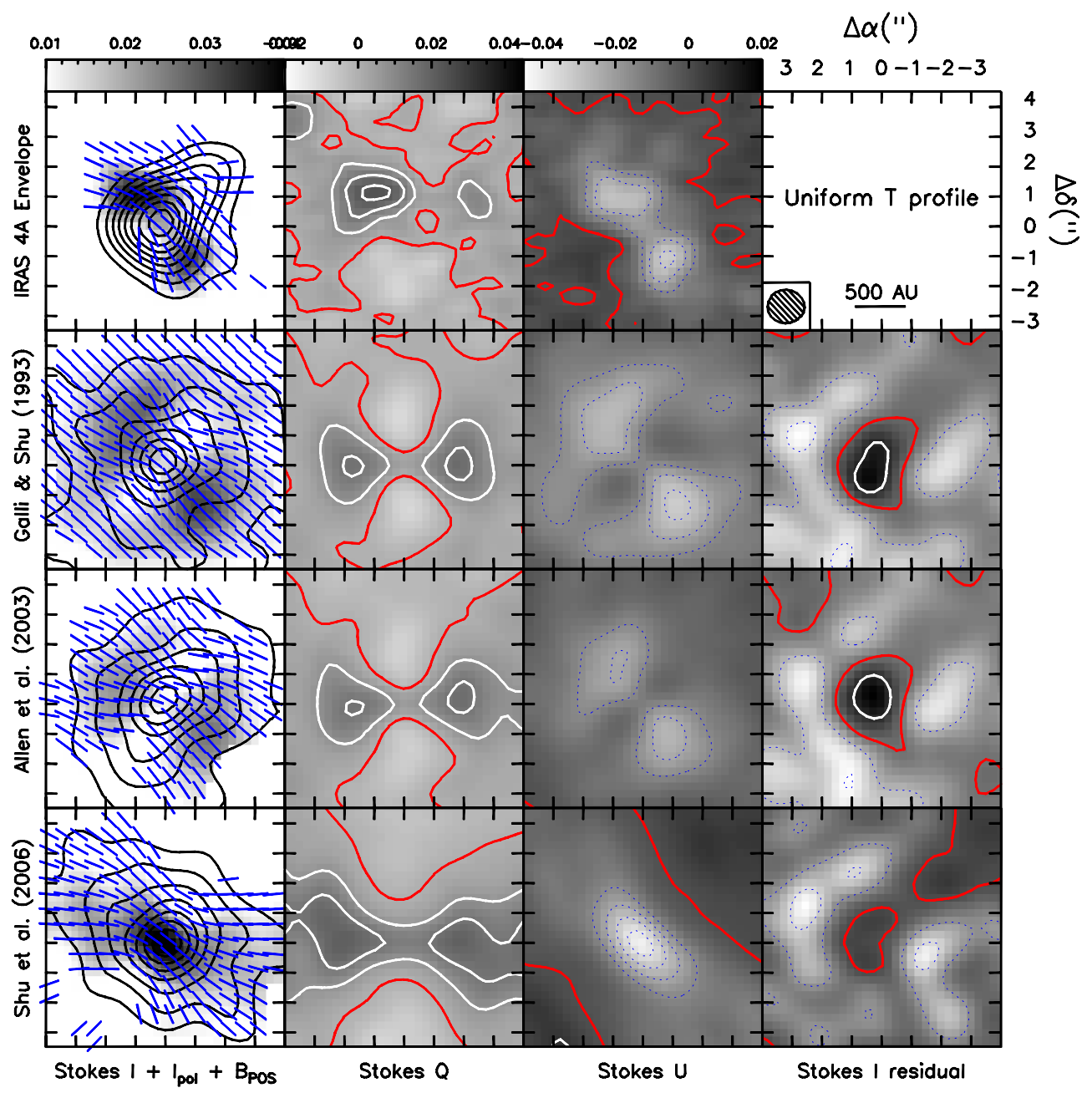

Fig. 7. Comparison of selected models with data assuming a uniform temperature of the gas. The orientation angles are fixed to $\phi=50^{\circ}$ and $\omega=45^{\circ}$ for a better model comparison. Rows: IRAS 4A (first row); model Galli \& Shu (1993a,b) with $B_{0}=0.43$ and $\tau=0.7$ (second row); model Allen et al. (2003a,b) with $H_{0}=0.125, v_{0}=0$ and $t=10^{4} \mathrm{yr}$ (third row); and model Shu et al. (2006) with $r_{\mathrm{Ohm}}=75$ AU (fourth row). Columns: in each row, the panels show: intensity (first panel, contours), polarized intensity (first panel, pixel map) and magnetic field vectors (first panel, segments); map of Stokes $Q$ (second panel, pixel map and contours); map of Stokes $U$ (third panel, pixel and contours); residuals models-data for Stokes $I$ (fourth panel, pixel map and contours). The color scale is shown on the top of each column. Contours: contours for the Stokes $I$ maps (left panels) depict emission levels from $6 \sigma$ up to the maximum value in steps of $6 \sigma$, where $\sigma=0.02 \mathrm{Jy} \mathrm{beam}^{-1}$. Coutours for the Stokes $Q$ and $U$ maps depict levels from the minimum up to the maximum in steps of $3 \sigma$ where $\sigma=2.5 \mathrm{mJy} \mathrm{beam}^{-1}$. The solid red contour marks the zero emission level, solid white contours mark positive emission and blue dotted contours mark negative emission. Contours for the residual Stokes $I$ follow the same rule of Stokes $Q$ and $U$ but with steps of $6 \sigma$, where $\sigma=0.02 \mathrm{Jy} \mathrm{beam}^{-1}$. The top right panel shows the beam and the angular and spatial scale.

Table 2. Models with uniform temperature profile: $880 \mu \mathrm{m}$ continuum emission and derived parameters ${ }^{a}$.

\begin{tabular}{lcccc}
\hline \hline & Unit & Galli \& Shu (1993a,b) & Allen et al. (2003a,b) & Shu et al. (2006) \\
\hline$S_{v}^{\mathrm{I}}$ & Jy & $8.92 \pm 0.25$ & $7.73 \pm 0.25$ & $8.35 \pm 0.25$ \\
$I_{\text {Peak }}^{\mathrm{I}}$ & $\mathrm{Jy} \mathrm{beam}^{-1}$ & $0.77 \pm 0.03$ & $0.79 \pm 0.03$ & $0.98 \pm 0.03$ \\
$F W H M^{b}$ & $\mathrm{AU}\left({ }^{\prime \prime}\right)$ & $919(3.06)$ & $1015(3.39)$ & $892(2.84)$ \\
\hline$S_{v}^{\text {pol }}$ & $\mathrm{mJy}$ & $390 \pm 40$ & $301 \pm 30$ & $328 \pm 30$ \\
$I_{\text {Peak }}^{\text {pol }}$ & mJy beam $^{-1}$ & $31 \pm 2$ & $34 \pm 2$ & $43 \pm 2$ \\
$\Omega^{\text {polc }}$ & arcsec $^{2}$ & 28 & 30 & 23 \\
\hline$\tau$ & - & 0.27 & 0.18 & 0.30 \\
$N_{\mathrm{H}_{2}}{ }^{d}$ & $10^{24} \mathrm{~cm}^{-2}$ & 4.61 & 3.14 & 5.11 \\
$n_{\mathrm{H}_{2}}{ }^{2}$ & $10^{8} \mathrm{~cm}^{-3}$ & 5.03 & 3.10 & 6.02 \\
$\mathrm{Mass}^{d}$ & $M_{\odot}$ & 1.92 & 1.60 & 1.82 \\
\hline
\end{tabular}

Notes. ${ }^{(a)}$ See Appendix A of Frau et al. (2010) for details. ${ }^{(b)}$ Diameter of the circle with the same area as the region of the source above the half peak intensity. ${ }^{(c)}$ Solid angle of the region with polarized intensity above $3 \sigma$. (d) Assuming $\kappa_{250 \mathrm{GHz}}=1.5 \mathrm{~cm}^{2} \mathrm{~g}^{-1}$ and a gas-to-dust ratio of 100 (Girart et al. 2006). 


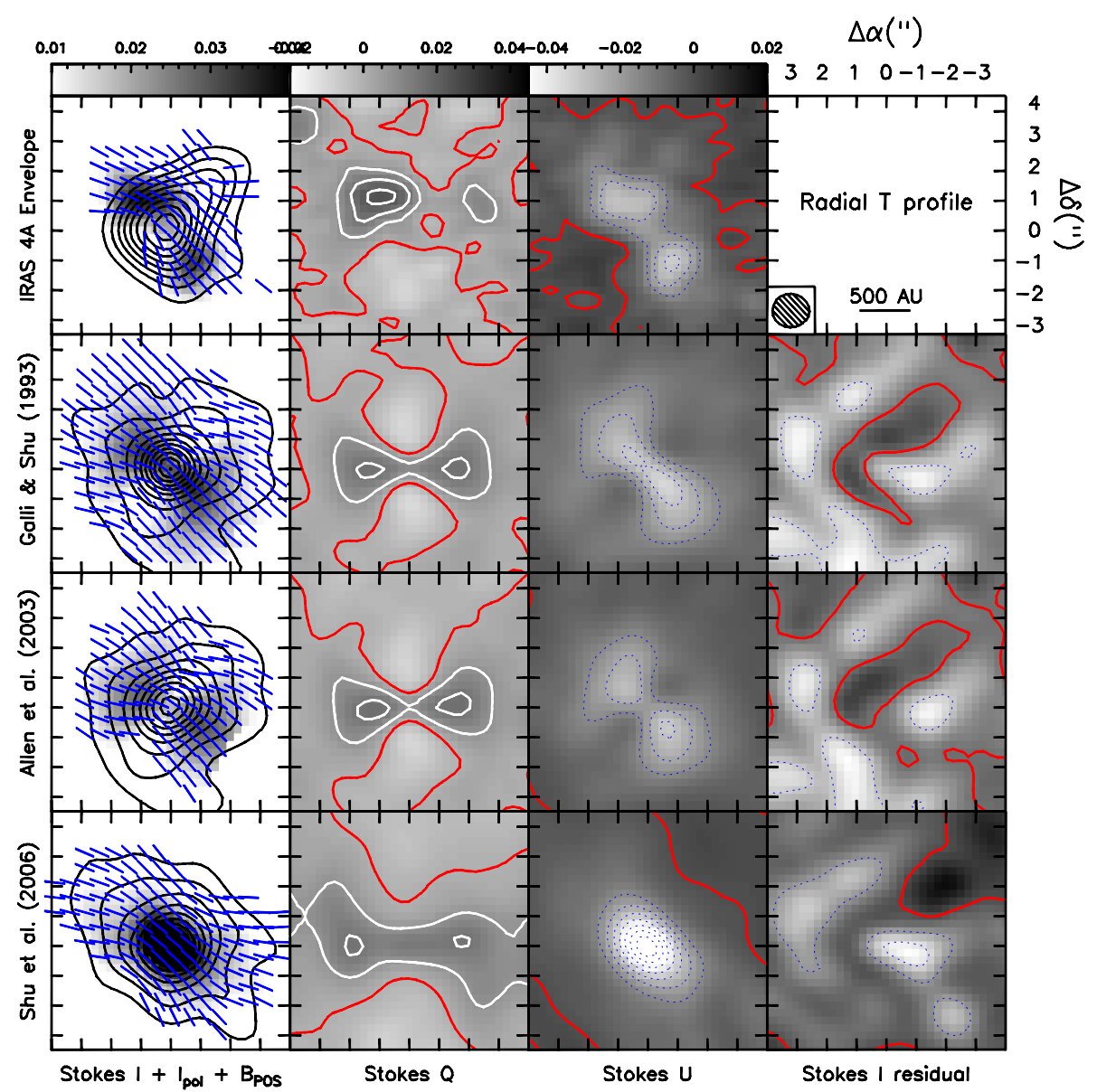

Fig. 8. Same as Fig. 7 for the case with the Maret et al. (2002) observational temperature gradient.

Table 3. Models with radial temperature profile: $880 \mu \mathrm{m}$ continuum emission and derived parameters ${ }^{a}$.

\begin{tabular}{lcccc}
\hline \hline & Unit & Galli \& Shu (1993a,b) & Allen et al. (2003a,b) & Shu et al. (2006) \\
\hline$S_{v}^{\mathrm{I}}$ & $\mathrm{Jy}$ & $6.59 \pm 0.25$ & $6.37 \pm 0.25$ & $6.90 \pm 0.25$ \\
$I_{\text {Peak }}^{\mathrm{I}}$ & Jy beam $^{-1}$ & $1.08 \pm 0.03$ & $1.02 \pm 0.03$ & $1.28 \pm 0.03$ \\
$F W H M^{b}$ & $\mathrm{AU}\left(^{\prime \prime}\right)$ & $693(2.31)$ & $744(2.48)$ & $693(2.31)$ \\
\hline$S_{v}^{\text {pol }}$ & $\mathrm{mJy}$ & $239 \pm 24$ & $173 \pm 17$ & $230 \pm 23$ \\
$I_{\text {Peak }}^{\text {pol }}$ & mJy beam $^{-1}$ & $32 \pm 2$ & $30 \pm 2$ & $68 \pm 2$ \\
$\Omega^{\text {polc }}$ & arcsec $^{2}$ & 16 & 12 & 12 \\
\hline$\tau$ & - & 0.37 & 0.30 & 0.39 \\
$N_{\mathrm{H}_{2}}{ }^{d}$ & $1{ }^{24} \mathrm{~cm}^{-2}$ & 6.28 & 5.12 & 6.64 \\
$n_{\mathrm{H}_{2}}{ }^{2}$ & $10^{8} \mathrm{~cm}^{-3}$ & 9.09 & 6.91 & 9.61 \\
Mass $^{d}$ & $M_{\odot}$ & 1.47 & 1.39 & 1.57 \\
\hline
\end{tabular}

Notes. ${ }^{(a)}$ See Appendix A of Frau et al. (2010) for details. ${ }^{(b)}$ Diameter of the circle with the same area as the region of the source above the half peak intensity. ${ }^{(c)}$ Solid angle of the region with polarized intensity above $3 \sigma .{ }^{(d)}$ Assuming $\kappa_{250} \mathrm{GHz}=1.5 \mathrm{~cm}^{2} \mathrm{~g}^{-1}$ and a gas-to-dust ratio of 100 (Girart et al. 2006).

gradient, respectively. The radial temperature profile realizations show a better match to the observational maps for all the Stokes maps, as well as physical parameters closer to the observed toward IRAS 4A. The general morphology of the magnetic field could be reproduced, as well as the depolarization toward the source axis and the double peak in polarized emission.

\subsection{Allen et al. (2003a,b)}

For the models of Allen et al. (2003a,b) we selected 3 nonrotating models with different values of the parameter $H_{0}$ defining the mass-to-flux ratio of the initial state $\left(H_{0}=0.125\right.$, 0.25 and 0.5 , corresponding to a mass-to-flux ratio in units of the critical value of $8.38,4.51$, and 2.66), and one rotating model, with $H_{0}=0.125$ and uniform rotation velocity $v_{0}=0.125$ (in units of the sound speed). For each case, we considered 5 evolutionary times, $t=10^{4} \mathrm{yr}, 2 \times 10^{4} \mathrm{yr}, 3 \times 10^{4} \mathrm{yr}, 4 \times 10^{4} \mathrm{yr}$ and $5 \times 10^{4} \mathrm{yr}$. As before, the position angles $\phi$ and $\omega$ were varied over a grid of $10 \times 7$ values, in both uniform and radial temperature profiles, generating a total of 2660 maps (due to numerical problems it was impossible to simulate the case $H_{0}=0.5$ with $\left.t=10^{4} \mathrm{yr}\right)$. The resulting $\chi^{2}$ of the comparison with the observed 
P. Frau et al.: Comparing star formation models with interferometric observations. I.



Fig. 9. Same as Fig. 3 for the Allen et al. (2003a,b) model with radial temperature profile.

Stokes $Q$ and $U$ maps for the radial temperature profile cases is shown in Fig. 9.

It is evident from the figure that better fits are obtained with lower values of the time elapsed since the onset of collapse, a few $10^{4} \mathrm{yr}$. The results are not very sensitive to the mass-to-flux ratio nor to the rotation of the initial configuration. There is a clear degeneracy between time and inclination angle with respect to the plane of the sky: a more concentrated field (a more pinched hourglass) can be obtained by letting the model evolve, or by a larger inclination of the magnetic field axis. For this reason, the region of minimum $\chi^{2}$ moves towards lower values of $\omega$ at later times.

Figures 7 and 8 show the predicted Stokes $I, Q, U$ maps and the Stokes $I$ residuals (third row) compared to the observed maps (first row) for this model, for the case with uniform temperature and temperature gradient, respectively. Both realizations shown have $H_{0}=0.125, v_{0}=0, t=10^{4} \mathrm{yr}, \phi=50^{\circ}$ and $\omega=45^{\circ}$. The derived physical parameters are shown in Tables 2 and 3 for the case with uniform temperature and temperature gradient, respectively. As for the Galli \& Shu (1993a,b) models, radial temperature profile realizations show a better match. For this model, the magnetic field morphology, source axis depolarization, and double peak in polarized emission could be reproduced like with the Galli \& Shu (1993a,b) models, although the polarized intensity derived was smaller in this case.

\subsection{Shu et al. (2006)}

To test this model, we varied the Ohm radius from $5 \mathrm{AU}$ to $150 \mathrm{AU}$. This parameter controls the size of the region where magnetic dissipation takes place and the magnetic field lines are almost straight. A total of 980 maps were generated, for 7 values of the Ohm radius, $10 \times 7$ values of the position and inclination angles, and both isothermal and radial temperature profiles. Figure 10 shows the $\chi^{2}$ of the comparison with the observed $Q$ and $U$ maps for the radial temperature profile case. The best-fit models tend to have $r_{\mathrm{Ohm}}$ in the range 10-100 AU, as also found previously (Gonçalves et al. 2008).

Figures 7 and 8 show the predicted Stokes $I, Q, U$ maps and the Stokes $I$ residuals (fourth row) compared to the observed maps (first row) for this model, for the case with uniform



Fig. 10. Same as Fig. 3 for the Shu et al. (2006) model with radial temperature profile.

temperature and temperature gradient, respectively. Both realizations shown have $r_{\mathrm{Ohm}}=75 \mathrm{AU}, \phi=50^{\circ}$ and $\omega=45^{\circ}$. The derived physical parameters are shown in Tables 2 and 3 for the case with uniform temperature and temperature gradient, respectively. As shown by the figure, this model fails to reproduce the double-peaked distribution of polarized intensity. This feature is associated to strongly concentrated, almost radial magnetic field lines in the central region, at variance with the almost uniform field morphology produced by magnetic dissipation. Another characteristic of this model is the relatively high degree of polarization, not supported by the observations.

\section{ALMA synthetic maps}

The ALMA resolution used $\left(0.7 \times 0.0^{\prime} 4 \sim 210 \times 120 \mathrm{AU}^{2}\right)$ was chosen to have several projected cells of the simulations ( $\sim 30$ AU) inside each beam, allowing the best comparison possible with the models avoiding resolution effects. The ALMA sensitivity and $u, v$ coverage is far much better than that of the SMA and, thus, allows ( $i$ ) to map with a much higher fidelity the polarized emission, and (ii) to detect emission from a larger and fainter region.

Figure 11 shows the ALMA maps for the models shown in Fig. 8 using the radial temperature profile. The level of detail of the convolved maps was very close to the original maps thus an almost perfect morphology reconstruction is possible for all the Stokes maps with the ALMA capabilities. Furthermore, the combination of sensitivity and resolution achievable with ALMA makes possible to extract usable information from the maps in a spatial range $\sim 10$ times larger than the resolution. We derived large and accurate polarization maps for all of the models. As Fig. 11 states, it will be possible with ALMA to reach resolution and detail levels which will allow to differentiate among different models, and to select those matching better the observations. In order to make this result more evident we marked in the left-hand side panel of each model a red circle depicting important distances related to the models. In the case of Galli \& Shu (1993a,b) and Allen et al. (2003a,b) red circles depict the loci of the isothermal collapse wave $\left(r=c_{\mathrm{s}} t\right)$ which can be compared directly to Figs. 2 and 6 of the original papers, respectively. For the Shu et al. (2006) model, red circle depict the $10 r_{\text {Ohm }}$ distance, comparable with Fig. 4 of their paper. The high power of reproduction of ALMA encourages polarization observation toward all of the sources as a detailed modeling will be possible with the onset of this powerful instrument. 




Fig. 11. Same as Fig. 8 for ALMA configuration 09. Contours: contours for the Stokes $I$ maps (left panels) depict emission levels from $5 \sigma$ up to the maximum value in steps of $50 \sigma$, where $\sigma=0.2 \mathrm{mJy} \mathrm{beam}^{-1}$. Positive contours for the Stokes $Q$ and $U$ maps depict levels from $5 \sigma$ up to the maximum in steps of $10 \sigma$, where $\sigma=0.02 \mathrm{mJy} \mathrm{beam}^{-1}$. Negative contours follow the same rule. Red circles: for the Galli \& Shu (1993a,b) and Allen et al. (2003a,b) models depict the loci of the front of the isothermal collapse wave $\left(r=c_{s} t\right.$, see Fig. 2 of Galli \& Shu 1993a and Fig. 6 of Allen et al. 2003a). For the Shu et al. (2006) model it marks the $10 r_{\text {Ohm }}$ distance (see Fig. 4 of Shu et al. 2006).

\section{Summary and conclusions}

The new data used in this work allowed to obtain a much better $u, v$ coverage for the IRAS $4 \mathrm{~A}$ region than in previous works, and, therefore, more reliable maps. In addition, the data added from different telescope configurations provided larger baselines, to resolve the compact components, as well as shorter baselines, to better trace the extended envelope emission. This significant improvement allowed to separate the embedded compact sources from the diffuse envelope. A good $u, v$ coverage was essential to perform reliable comparisons with models. To this goal, we developed a selection method, the so-called $\chi^{2}$ method, with larger discriminating power than in previous studies (e.g., Gonçalves et al. 2008).

The new data confirm that the source emission is optically thin, with no absorption or scattering, as expected for sub-mm emission. The opacity derived from the Stokes $I$ map of the envelope is negligible, implying that the maps trace very deep into the source. As the scattering appear to be negligible, the origin of the alignment of the dust grains is expected to be due to the magnetic field.

Despite the complexity of the NGC 1333 star forming region, MHD models of single star formation assuming quasi-static initial conditions and a uniform (or nearly uniform) magnetic field show remarkable agreement with the observed characteristics of IRAS 4A, like intensity, polarized intensity, and polarization distribution. These facts suggests that the dust polarization pattern resulting from the density and magnetic field distribution of non-turbulent models may apply even in less idealized initial conditions than normally assumed. Once the orientation angles are consistently determined, the comparison of the data with models of magnetized collapse indicate that a strong initial field is required $\left(B_{0}\right.$ larger than a few tenths of $\mathrm{mG}$, see Sect. 3.1), that the source is very young (a few $10^{4} \mathrm{yr}$, see Sects. 3.1 and 3.2), and that the scale where magnetic dissipation occurs is below the resolution of current observations (see Sect. 3.3). However, with the current level of sensitivity and $u, v$ coverage it is not possible to clearly discriminate among different collapse models. The Galli \& Shu (1993a,b) and Allen et al. (2003a,b) models fit better than the Shu et al. (2006) model, but no selection can be done between the former two models.

In general, the models predict sources with a more centrally peaked core and a larger less dense envelope with polarized emission less concentrated. A more refined dust grain treatment could help in a more realistic emission treatment. Current SMA observations of IRAS 4A clearly favor models 
with a temperature gradient. The total emission maps derived from models with a temperature gradient show the right peak value but larger fluxes, steeper profiles and more extended morphologies than IRAS 4A. On the other hand, they predict the right fluxes and morphologies of polarized emission but lower peaks and softer profiles. Conversely, polarization maps obtained with uniform temperature profiles are more extended than IRAS 4A maps (see Sect. 5.2). Therefore, the inclusion of a realistic temperature profile cannot be ignored in modeling the sub-mm emission of low-mass protostars.

An important result from the simulations of protostellar enveloped threaded with an hourglass magnetic field is the possibility of deriving the orientation angles of real sources from polarization measurements. Figures 4 and 5 show that up to inclination angles of $\sim 60^{\circ}$ it is possible to estimate both position and inclination angles from the Stokes $Q$ and $U$ maps. For larger inclinations the magnetic field tends to be radial and the Stokes maps do not show significant differences. Therefore, Figs. 4 and 5 can be used as templates for future observations of the dust polarized emission toward star forming cores.

Another remarkable result is the good agreement in the $u, v$-plane of the observed and synthetic visibility amplitudes obtained assuming a temperature gradient, except for the shortest baselines $(\$ 20 \mathrm{k} \lambda)$. The observed deficit of emission in IRAS 4A with respect to the models suggest a sharper density decrease at scales of $\sim 1500$ AU than predicted by theoretical models.

The ALMA simulations have shown the capability of this new instrument to distinguish fine details even between models of the same family. The methodology used in this work has proved to be a powerful tool to compare observations directly to theoretical models in a consistent way and avoiding instrumental effects. Future polarization measurements with the ALMA will provide real power to select the best models to describe the structure and evolution of low-mass cores and, consequently, to disentangle the medium conditions and the physics ruling the process. Upcoming radiative transfer codes like ARTIST (Padovani et al. 2011) will facilitate this kind of studies. ALMA data, together with powerful radiative transfer codes, may be used together with the technique developed in this work to extract as much information as possible from the data and to constrain the models.

Acknowledgements. P.F. is partially supported by MICINN grant FPU. P.F. and J.M.G. are supported by MICINN grant AYA2008-06189-C03. P.F. and J.M.G. are also supported by AGAUR grant 2009SGR1172. The authors are grateful to Shih-Ping Lai and Tao-Chung Ching for gently sharing their SMA data. The authors also thank José Gonçalves and Jongsoo Kim for useful discussions, and the anonymous referee for useful comments.

\section{References}

Allen, A., Shu, F. H., \& Li, Z.-Y. 2003a, ApJ, 599, 351

Allen, A., Li, Z.-Y., \& Shu, F. H. 2003b, ApJ, 599, 363

Blake, G. A., Sandell, G., van Dishoeck, E. F., et al. 1995, ApJ, 441, 689

Briggs, D. S. 1995, BAAS, 27, \#112.02

Chandrasekhar, S., \& Fermi, E. 1953, ApJ, 118, 113

Choi, M. 2005, ApJ, 630, 976

Crutcher, R. M., Hakobian, N., \& Troland, T. H. 2009, ApJ, 692, 844

Di Francesco, J., Myers, P. C., Willner, D. J., Ohashi, N., \& Mardones, D. 2001, ApJ, 562, 770

Frau, P., Girart, J. M., Beltrán, M. T., et al. 2010, ApJ, 723, 1665

Fiedler, R. A., \& Mouschovias, T. C. 1993, ApJ, 415, 680

Fiege, J. D., \& Pudritz, R. E. 2000, ApJ, 544, 830

Galli, D., \& Shu, F. H. 1993a, ApJ, 417, 220

Galli, D., \& Shu, F. H. 1993b, ApJ, 417, 243

Galli, D., Lizano, S., Shu, F. H., \& Allen, A. 2006, ApJ, 647, 374

Girart, J. M., Crutcher, R. M., \& Rao, R. 1999, ApJ, 525, L109

Girart, J. M., Rao, R., \& Marrone, D. P. 2006, Science, 313, 812

Gonçalves, J., Galli, D., \& Walmsley, M. 2005, A\&A, 430, 979

Gonçalves, J., Galli, D., \& Girart, J. M. 2008, A\&A, 490, L39

Hildebrand, R. H. 1983, QJRAS, 24, 267

Jennings, R. E., Cameron, D. H. M., Cudlip, W., \& Hirst, C. J. 1987, MNRAS, 226, 461

Lai, S.-P., Crutcher, R. M., Girart, J. M., \& Rao, R. 2002, ApJ, 566, 925

Lay, O. P., Carlstrom, J. E., \& Hills, R. E. 1995, ApJ, 452, L73

Lazarian, A. 2003, J. Quant. Spec. Radiat. Transf., 79, 881

Lee, H. M., \& Draine, B. T. 1985, ApJ, 290, 211

Lee, J.-E., \& Kim, J. 2009, ApJ, 699, L108

Li, Z.-Y., \& Shu, F. H. 1996, ApJ, 472, 211

Looney, L. W., Mundy, L. G., \& Welch, W. J. 2000, ApJ, 529, 477

Maret, S., Ceccarelli, C., Caux, E., Tielens, A. G. G. M., \& Castets, A. 2002, A\&A, 395, 573

Mouschovias, T. C., \& Spitzer, L., Jr. 1976, ApJ, 210, 326

Myers, P. C., \& Goodman, A. A. 1991, ApJ, 373, 509

Nakamura, F., \& Li, Z.-Y. 2005, ApJ, 631, 411

Nakano, T., \& Nakamura, T. 1978, PASJ, 30, 671

Novak, G., Gonatas, D. P., Hildebrand, R. H., Platt, S. R., \& Dragovan, M. 1989, ApJ, 345, 802

Ostriker, E. C., Stone, J. M., \& Gammie, C. F. 2001, ApJ, 546, 980

Padoan, P., Goodman, A., Draine, B. T., et al. 2001, ApJ, 559, 1005

Padovani, M., Jorgensen, J. K., Bertoldi, F., et al. 2011, IAU Symp., 270, 451

Rao, R., Girart, J. M., Marrone, D. P., Lai, S.-P., \& Schnee, S. 2009, ApJ, 707, 921

Ridge, N. A., Schnee, S. L., Goodman, A. A., \& Foster, J. B. 2006, ApJ, 643, 932

Sandell, G., Aspin, C., Duncan, W. D., Russell, A. P. G., \& Robson, E. I. 1991, ApJ, 376, L17

Shu, F. H., Galli, D., Lizano, S., \& Cai, M. 2006, ApJ, 647, 382

Strittmatter, P. A. 1966, MNRAS, 132, 359

Tang, Y.-W., Ho, P. T. P., Koch, P. M., et al. 2009, ApJ, 700, 251

Tomisaka, K., Ikeuchi, S., \& Nakamura, T. 1988, ApJ, 335, 239

Vázquez-Semadeni, E., Kim, J., Shadmehri, M., \& Ballesteros-Paredes, J. 2005, ApJ, 618, 344

Wardle, M., \& Konigl, A. 1990, ApJ, 362, 120

Zweibel, E. G. 1990, ApJ, 362, 545 